LBL-32829

\title{
Electronic Structure of Substitutionally Disordered Alloys: Direct Configurational Averaging
}

\author{
C. Wolverton \\ LBL- -32829 \\ Department of Physics \\ University of California \\ and \\ Materials Sciences Division \\ Lawrence Berkeley Laboratory \\ Berkeley, California 94720
D. de Fontaine
Department of Materials Science and Mineral Engineering
University of California
and
Materials Sciences Division
Lawrence Berkeley Laboratory
Berkeley, California 94720 \\ H. Dreysse \\ Laboratoire de Physique du Solide \\ Universite de Nancy \\ Laboratoire de Physique du Solide \\ Vandoeuvre-les-Nancy, France \\ and \\ G. Ceder \\ Department of Materials Science \\ Massachusetts Institute of Technology \\ Cambridge, Massachusetts
}

April 1992

\begin{abstract}
This work was supported by the Director, Office of Energy Research, Office of Basic Energy Sciences, Materials Sciences Division, of the U.S. Department of Energy under Contract No. DE-AC03-76SF00098, by a grant from NATO (\#0512/88), and by a grant from the CNRS-NSF (INT-8815493). One of the authors (DdF) would also like to acknowledge the support of the Advanced Materials and Technology Laboratory of the Nippon Steel Corporation.
\end{abstract}


ELECTRONIC STRUCTURE OF SUBSTITUTIONALLY DISORDERED ALLOYS: DIRECT CONFIGURATIONAL AVERAGING

\section{WOLVERTON ${ }^{1}$, D. DE FONTAINE ${ }^{2}$, H. DREYSSE ${ }^{3}$, AND G. CEDER $^{4}$}

1 Department of Physics, University of Califomia at Berkeley (UCB), and Lawrence Berkeley Laboratory (LBL), Berkeley, CA.

2Department of Materials Science and Mineral Engineering, UCB, and LBL, Berkeley, CA.

${ }^{3}$ Laboratoire de Physique du Solide, Universite de Nancy, Laboratoire de Physique du Solide, Vandoeuvre-les-Nancy, France.

${ }^{4}$ Department of Materials Science, Mass. Institute of Technology, Cambridge, MA.

\section{ABSTRACT}

The method of direct configurational averaging (DCA) has been proposed to study the electronic structure of disordered alloys. Local density of states and band structure energies are obtained by averaging over a small number of configrations within a tight-binding Hamiltonian. Effective cluster interactiors, the driving quantities for ordering in solids, are computed for various alloys using a tight-oinding form of the linearized muffin-tin orbital method (TB-LMTO). The DCA calculations are used to determine various energetic and thermodynamic quantities for binary and ternary alloys.

\section{INTRODUCTION}

The ability to predict structural, electronic, and thermodynamic properties of alloys is of great scientific and technological importance. In the past, only empirical approaches have been used to study alloy systems. However, recent advances in first-principles electronic band structure calculations based on the local density approximation now make possible the theoretical computation of lattice constants, bulk modulii, cohesive energy, etc. in good agreement with the experimentally measured quantities. Most of these methods only treat perfectly ordered, stoichiometric compounds at zero temperature. In order to understand the properties of alloys, it is necessary to predict the energetics of disordered, partially ordered, and off-stoichiometric structures both at zero and finite temperatures. Thus, a thermodynamic treatment must be combined with the zero temperature electronic structure calculations, and the electronic structure must be expanded to provide reliable expressions for the configurational energy.

The formulation of this problem is facilitated by mapping the alloy onto an extended "Ising. like" model. Thus, for a binary alloy, each site in the lattice is associated with a "pseudo-spin" variable, $\sigma_{i}$, which is given the value $+1(-1)$ if an $A(B)$ atom is located at site $i$. Thus, any configuration of the lattice, $\sigma$, may be represented by the $\mathrm{N}$-dimensional vector, $\sigma=\left(\sigma_{1}, \sigma_{2}, \ldots, \sigma_{N}\right)$, where $N$ is the number of sites in the system. Sanchez, Ducastelle, and Gratias ${ }^{1}$ have shown that within this model, any function of configuration may be expanded in terms of cluster functions, or products of the spin variables. This type of expansion is exact, and in particular, one such quantity which may be expanded is the internal energy:

where

$$
\mathrm{E}(\sigma)=\mathrm{V}_{0}+\sum_{\alpha} \mathrm{V}_{\alpha} \sigma_{\alpha}
$$

$$
\sigma_{\alpha}=\left\langle\sigma_{1} \sigma_{2} \ldots \sigma_{n_{\alpha}}\right\rangle
$$

and the generalized Fourier coefficients, $V_{\alpha}$, are commonly refered to as effective cluster interactions (ECI's). These ECI's are given a precise definition within the context of the expansion. For the case of an effective pair interaction,

$$
V_{i j}=\frac{1}{4}\left[\left\langle E_{A A}\right\rangle+\left\langle E_{B B}\right\rangle-\left\langle E_{A B}\right\rangle-\left\langle E_{B A}\right\rangle\right]
$$

where $\left\langle E_{A B}\right\rangle$ is the average energy of all configurations with an $A-B$ pair at sites $i, j$.

In order to calculate these ECI's for a specific alloy system, then, the most straightforward method would involve explicitly computing the quantities in Eq. (3). However, this direct 
approach involves several difficulties: 1) There are $2^{\mathrm{N}-2}$ terms in each of the averages implied by $\left\langle E_{U}\right\rangle$, and in the thermodynamic limit of $\mathrm{N} \rightarrow \infty$, this clearly makes an exact calculation of all of these terms impossible. 2) Also, these averages involve all configurations on the lattice, and only an infinitesmally small number of these configurations possess some form of translational invariance. Thus, the standard techniques of band structure based of Bloch's theorem are not applicable to these completely disordered configurations. 3) The four terms in Eq. (3) involve energies of configurations which differ from one another by only one or two atoms. Therefore, an obvious practical difficulty of separately computing each term involves the determination of the small difference (the ECI) of large numbers (the energy of a given configuration). The method of direct configurational averaging (DCA), which is described below, overcomes all three of these obstables, while maintaining the desirable property of calculating the ECI's from Eq. (3), which is known to be exact.

\section{DIRECT CONFIGURATIONAL AVERAGING (DCA)}

Conceptually, the idea of DCA is essentially to compute the interactions directly from their definitions, as given in Eq. (3). DCA is described in detail by Dreysse ${ }^{2}$, but for completeness, we give the essential points here. First, a finite lattice of points is constructed in real space, and is decorated with $A$ and $B$ atoms in a given configuration. The Hamiltonian for this configuration is tridiagonalized using the recursion method ${ }^{3}$. Because this method is formulated in real space, the decorated lattice may have any configuration, i.e., the configuration need not be constrained by any symmetries. Given the Hamiltonian in tridiagonal form, the local density of states may be obtained as a continued fraction. The recursion must be stopped at some stage, and hence the continued fraction terminated. For this purpose, we use a quadratic terminator. Also, we find that ten levels of recursion is adequate for convergence of the ECI's.

Integrating the local density of states to the Fermi energy gives the band structure term of the cohesive on a given atom in any configuration. Thus, in order to calculate one of the terms in the definition of the $E C I,\left\langle E_{A B}>\right.$, for example, one may simply dress the lattice with $A$ and $B$ atoms at sites $i$ and $j$ respectively, with the rest of the lattice being decorated at random. Then, using recursion, one calculates $\mathrm{E}_{\mathrm{AB}}$ for a given configuration (not the averaged, $\left\langle\mathrm{E}_{\mathrm{AB}}>\right.$ ). In this way, it would be possible to obtain all the terms in Eq. (3). However, computing each of the terms in Eq. (3) separately and then taking differences is difficult due to large subtractive cancellations. The formalism of "orbital peeling"4 may be applied to the problem of computing the ECI's. Using this method, one may obtain the differences in Eq. (3) directly, and thus bypass the numerical instabilities caused by simple subtractions.

Thus, the averaging procedure involved in DCA is to calculate the differences in energies involved in the ECI's for a given (randomly generated) configuration. Then, in order to obtain the exact expression for the EPI given by Eq. (3), it would be necessary, in principle, to average this energy difference over all the possible configurations of the lattice. Fortunately, however, extensive calculations ${ }^{2}$ have shown that there is an extremely fast convergence of the ECI's with respect to the number of configurations visited. Therefore, in order to obtain a given ECI within any threshold of its exact value, one need only average over a finite set of configurations, the number of which, of course, being dictated by the accuracy required.

\section{RESULTS}

LMTO calculations were performed for $\mathrm{Pd}, \mathrm{Rh}$, and $\mathrm{V}$, all in the fcc structure. The calculations were done in the atomic sphere approximation. Scalar relativistic effects are included, and in the results for the ternary system, Pd-Rh-V, to be discussed below, combined corrections terms were included. The total energies of the pure elements were minimized with respect to their lattice constants. Then, a linear variation of alloy volume with respect to alloy composition was assumed, and the LMTO calculations were repeated for each of the constituents at the alloy volume. By the screening tranformation of the LMTO Hamiltonian 5 , the tight-binding representation of the pure elements was determined.

The matrix elements of the Hamiltonian change upon alloying, and this is modeled as a shift between the on-site energies of the two metals. This shift is determined self-consistently along with the Fermi energy by imposing local neutrality, a reasonable approximation for a transition 
metal alloy. Off-diagonal disorder is treated within the well established approximation of the hopping between unlike atoms being given by the geometric mean of the hopping integrals between like pairs.

The DCA was used, with the TB-LMTO, to compute the ECI's for three binary systems, Pd$\mathrm{V}, \mathrm{Rh}-\mathrm{V}, \mathrm{Pd}-\mathrm{Rh}$, and the ternary system bounded by these binaries, Pd-Rh-V. For each of the binary systems, the first through fcurth nearest neighbor pair interactions as well as several triplet and quadruplet interactions were calculated. First through fourth nearest neighbor pairs were calculated for the ternary system. The energy expansion of Eq. (1) is seen to be rapidly convergent as the pair interactions are seen to rapidly decay for each of the systems investigated. Also, the multiplet interactions rapidly decay as the number of points in the cluster is increased.
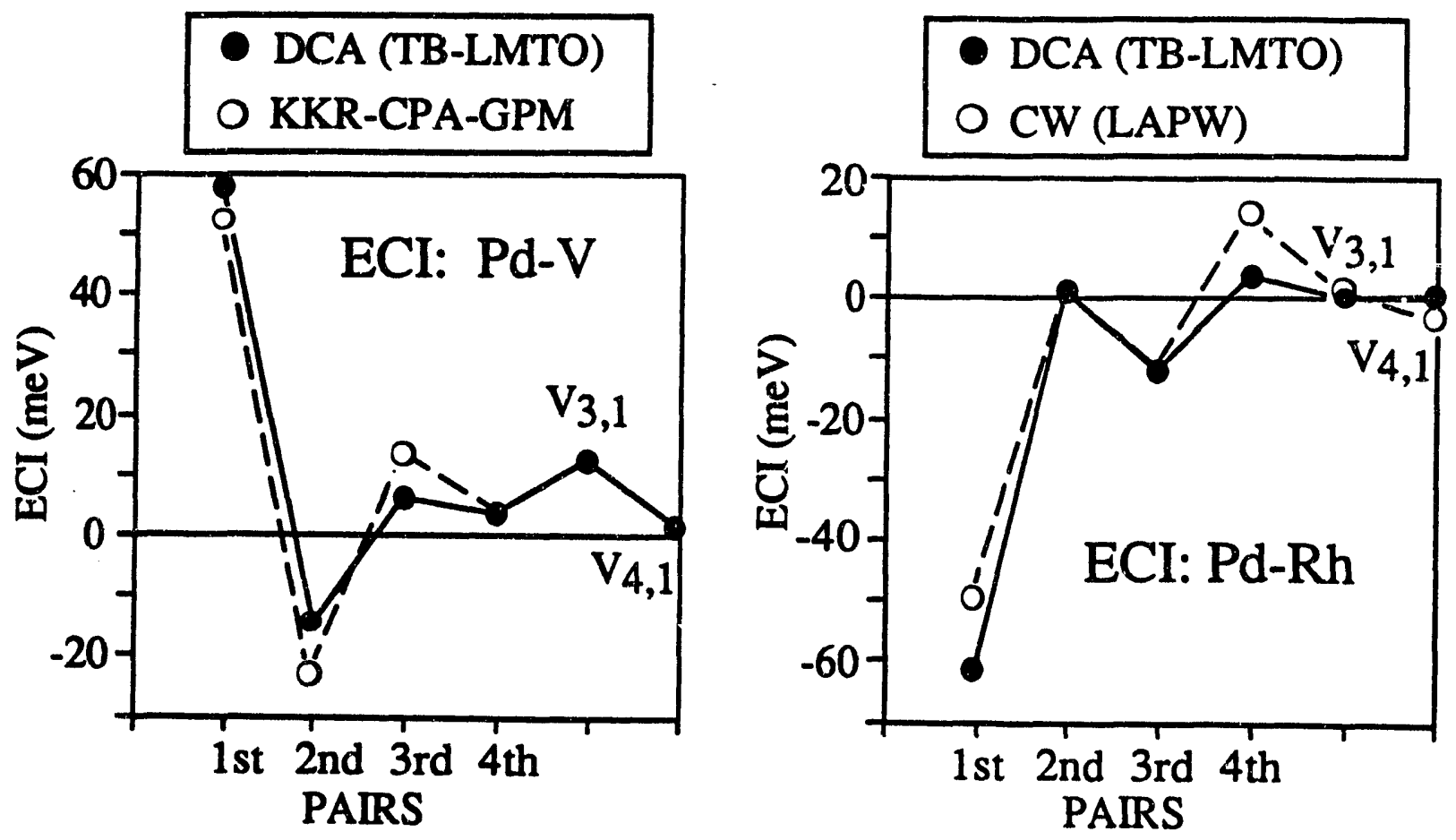

Fig. 1. Comparison of ECrs calculated from the DCA and those of other methods. The Pd-V results are compared with the pair interactions of Ref. 6 computed via the KKR-CPA-GPM. ECI's for the Pd-Rh system are compared with the results ${ }^{7}$ of the Connolly-Williams method using total energy, LAPW calculations. In both cases, the DCA (TB-LMTO) results are plotted as filled circles connected by solid lines. Empty circles connected by dashed lines denote the interactions of the other theories.

In Fig. 1, the interactions computed with the DCA are compared with those of other ab-initio methods of extracting the ECIs. In the Pd-V system, the comparison between the TB-LMTODCA interactions and those of ref. (6) is well within $10 \mathrm{meV}$ for each of the pair interactions. In addition to the pair interactions, the triplet and quadruplet interactions corresponding to the three and four-body clusters made up of only nearest neighbor bonds are also plotted. In the case of the $\mathrm{Pd}-\mathrm{Rh}$ system, comparison is made with another method which is based on linearized augmented plane wave calculations (LAPW) of ordered structures?. The agreement is quite good for both systems.

The formulation of the alloy problem on a-fixed "Ising-like" lattice allows for the exact determination of the ground state structures as. a function of composition for certain sets of interactions. Previously, these exact ground state searches did not include both interactions of fourth nearest neighbor extent and multiplets. However, both these types of interactions are known to be crucial in obtaining a converged energy expression. Recently, though, an exact ground state search on the fcc lattice has been performed with just such sets of interactions, the interactions being computed from the DCA ${ }^{8}$. Fig. 2 shows two such examples of these ground state searches. The ground state formation energies (simply the energies of the given structure with the concentration-weighted average of the pure element energies subtracted off) of both the $\mathrm{Pd}-\mathrm{V}$ and $\mathrm{Rh}-\mathrm{V}$ systems are shown as computed from the DCA-interactions. The stable phases must be connected by a convex hull, and this hull is shown as a solid line in the figure. Also, the 
formation energy of the completely random state is also shown as a dashed line. Although the Pd-V and Rh-V systems look very similar qualitatively (note the scale of the two plots is identical), the ground state structures are quite different. The ground state search has also been performed on the Pd-Rh system, but the results are trivial, as this system phase separates, and thus, the ground states are simply pure $\mathrm{Pd}$ and pure $\mathrm{Rh}$. In both cases, all of the experimentallyobserved fcc structures are predicted by the ground state search.
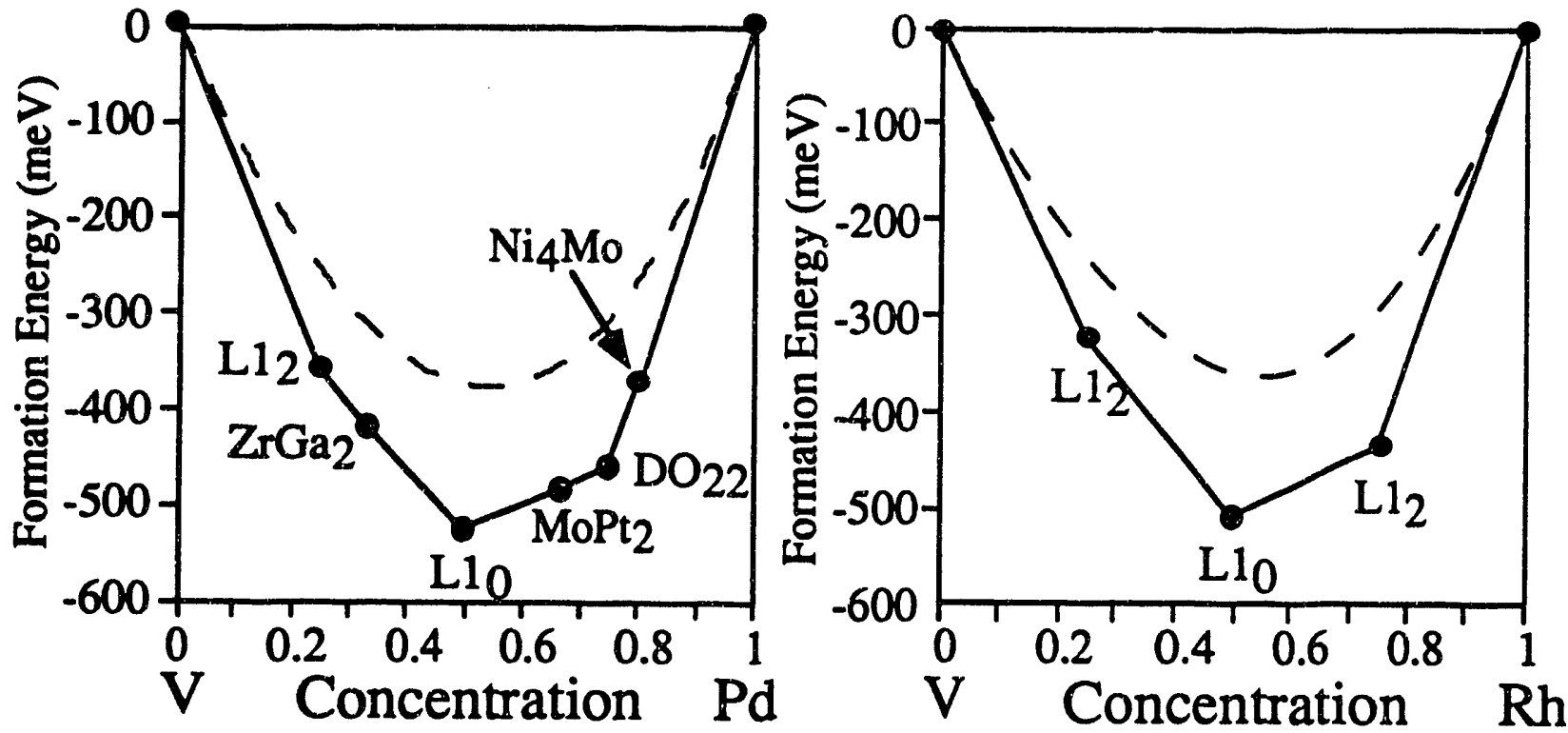

Fig. 2. Results of the fcc ground state search for the Pd-V and Rh-V systems. The stable phases, indicated by filled squares, are connected by tie lines. The formation energy of the completely random state is indicated as a function of concentration by a dashed curve.

Using the energy expansion of Eq. (1) in conjunction with the cluster variation method, it is possible to compute composition-temperature phase diagrams for these binary alloys with no adjustable parameters of the theory. Fig. 3 shows the result of such a calculation for the $\mathrm{Pd}-\mathrm{Rh}$ system. Only the atomic numbers, lattices, and lattice constants were used as input to the calculation. It is seen in this calculation that the correct treatment of off-diagonal disorder is crucial to obtaining good agreement with experimental results. Overestimating the off-diagonal

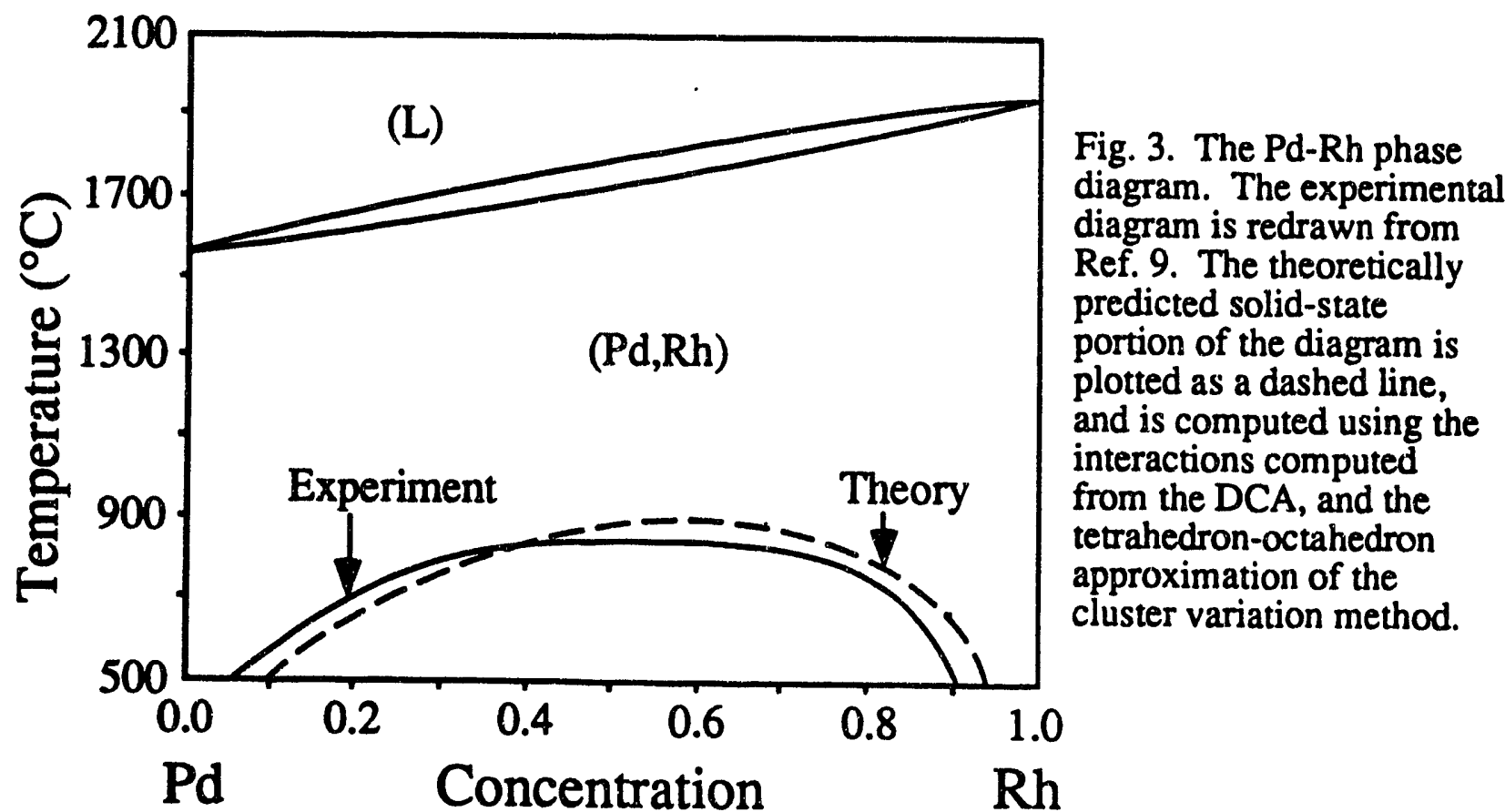


disorder leads to theoretically predicted phase diagrams with transition temperatures much higher than that of the experimental diagram. In Fig. 3, only the nearest neighbor and next nearest neighbor pairs were retained in the energy expansion for the calculation. However, in order to correctly predict the asymmetry of the phase diagram, it was necessary to compute the pair interaction separately for several values of the alloy volume. Then, the alloy volume was assumed to behave linearly with concentration in order to obtain the solid-state portion of the theoretically predicted diagram.

Recently, the DCA has been extended to study ternary alloys. In any theoretical study of a ternary alloy system, it is necessary to first determine that the theory correctly predicts the trends of the three binary systems. From the above discussion, it is apparent that the DCA combined with TB-LMTO should provide some insight into the Pd-Rh-V ternary system. In Fig. 4, the formation energies of the completely random state are plotted for this system. In a ternary system, the composition space is two-dimensional, and is usually shown in the form of a Gibbs triangle. The vertices of the triangle represent the three pure elements, and the sides of the triangle indicate the three binary systems. In Fig. 4 it can be seen that the Pd-Rh system has a small, but positive formation energy (consistent with this system's phase separating tendencies), and the Pd-V and Rh-V systems both strongly order. Although the formation energy of the random state becomes negative for small amounts of $\mathrm{V}$ added to an equiatomic Pd-Rh alloy, we shall see below that this does not, in fact, imply that $\mathrm{Pd}$ and $\mathrm{Rh}$ atoms develop ordering tendencies at these compositions.

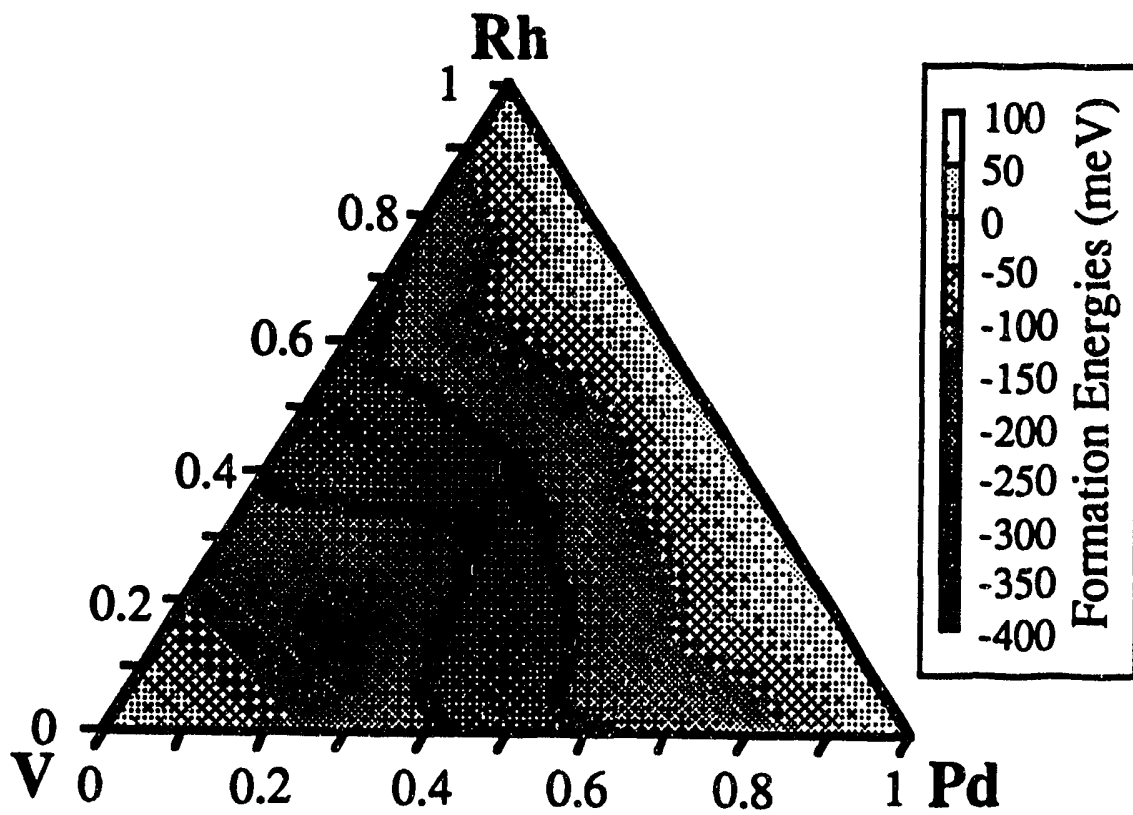

Fig. 4. Formation energy of the completely random state for the temary system Pd-Rh-V. The largest negative formation energies are seen to occur not at equi-atomic composition, but rather at two positions near the equi-atomic binary Rh-V and Pd-Vsystems.

Fig. 5 shows the three types of pair interactions for the Pd-Rh-V system plotted as a function of the alloy composition. It can be seen that although the quantitative value of the pair interaction may change significantly as a function of alloy composition (note particularly the strong composition-dependence of the $\mathrm{Pd}-\mathrm{V}$ interaction), the qualitative behavior remains the same. For example, the $\mathrm{Pd}-\mathrm{Rh}$ pair interaction indicates that it is energetically favorable for $\mathrm{Pd}$ and $\mathrm{Rh}$ to phase separate over the entire composition range of the alloy. Thus, the negative formation energy for alloys of Pd-Rh with small amounts of $\mathrm{V}$ added may be explained by the fact that many $\mathrm{Rh}-\mathrm{V}$ and $\mathrm{Pd}-\mathrm{V}$ bonds are created in this alloy, and because these pairs of atoms strongly favor ordering tendencies, this overcomes the weak positive formation energy of the Pd-Rh alloys.

\section{CONCLUSIONS}

The DCA has been used to calculate a variety of properties of binary and ternary alloys. It is seen that DCA, used in conjunction with the TB-LMTO provides a reliable, quantitative tool for the study of transition metal alloys. ECI's for several alloy systems have been computed and are in agreement with those of other theoretical predictions. These interaction also correctly predict the ground state properties of the $\mathrm{Pd}-\mathrm{V}$ and $\mathrm{Rh}-\mathrm{V}$ systems, as well as the phase diagram of the 
Pd-Rh system. The flexibility of the method has been illustrated in the extension to ternary alloy systems.

\section{ACKNOWLEDGEMENTS}

Research supported by the Director, Office of Basic Energy Sciences, Materials Science Division, of the U.S. Dept. of Energy under Contract No. DE-AC03-76F00098, by a grant from NATO (\#0512/88), and by a grant from the CNRS-NSF (INT-8815493). One of the authors (DdF) would also like to acknowledge the support of the Advanced Materials and Technology Research Laboratory of the Nippon Steel Corporation.

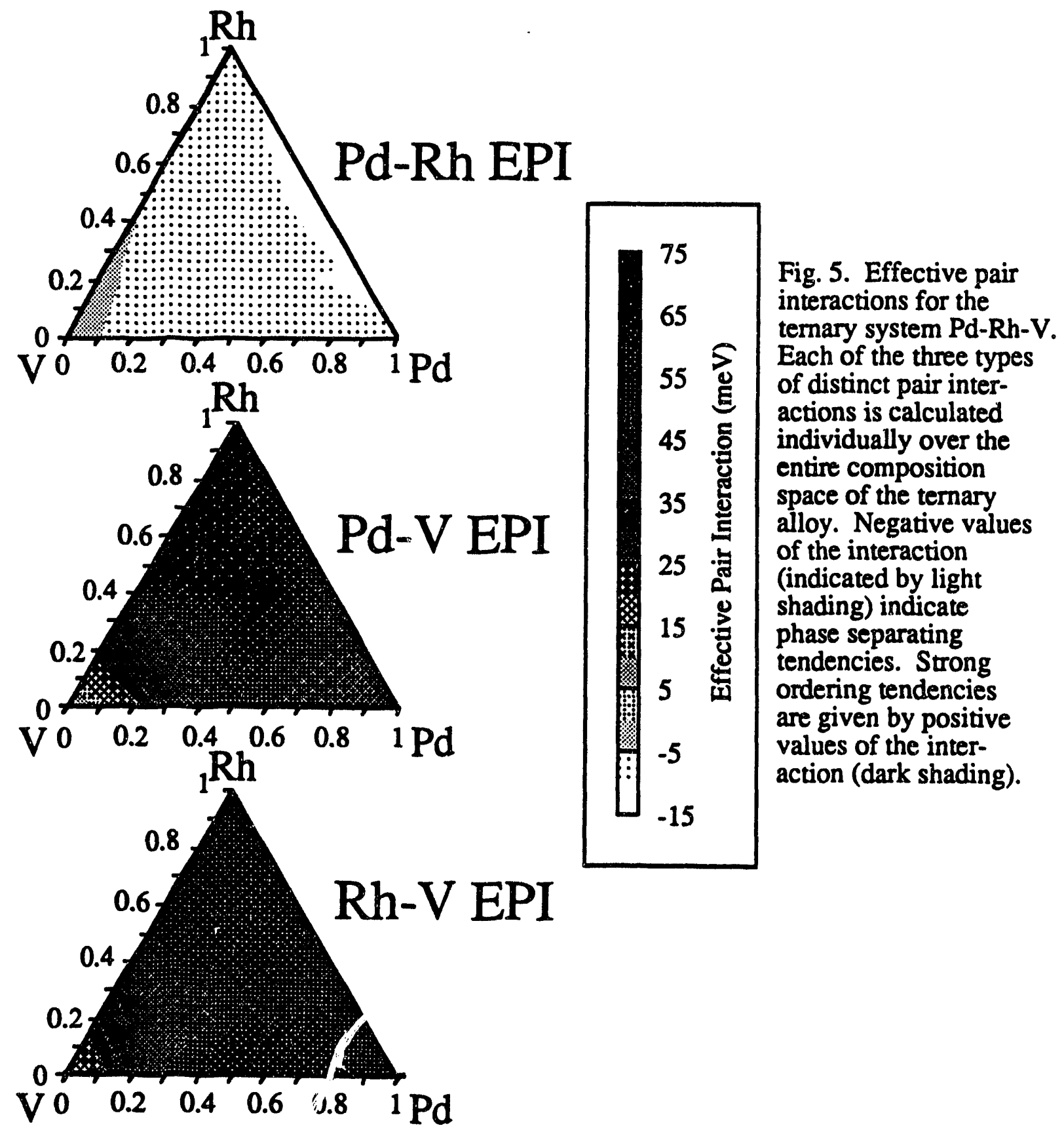

\section{REFERENCES}

1. J. M. Sanchez, F. Ducastelle, and D. Gratias, Physica A 128, 334 (1984).

2. H. Dreyssé, A. Berera, L. T. Wille, and D. de Fontaine, Phys. Rev. B39, 2442 (1989).

3. R. Haydock, Solid State Phys. 35, 215 (1980). 
4. N. R. Burke, Surf. Sci. 58, 349 (1976).

5. O. K. Andersen, O. Jepsen, and M. Sob, Electronic Band Structure and Its Applications, edited by M. Yussouf (Springer, Berlin, 1987).

6. P. Turchi, G. Stocks, W. Butler, D. Nicholson, and A. Gonis, Phys. Rev. B37, 5982 (1988).

7. Z. W. I u, S. -H. Wei, A. Zunger, and L. G. Ferreira, Solid State Commun. 78, 583 (1991).

8. C. Wolve;ton, G. Ceder, D. de Fontaine, and H. Dreysse, Phys. Rev. B45 (in press).

9. R.P. Elliot, Constitution of Binary Alloys, First Supplement, McGraw-Hill, New York (1965). 

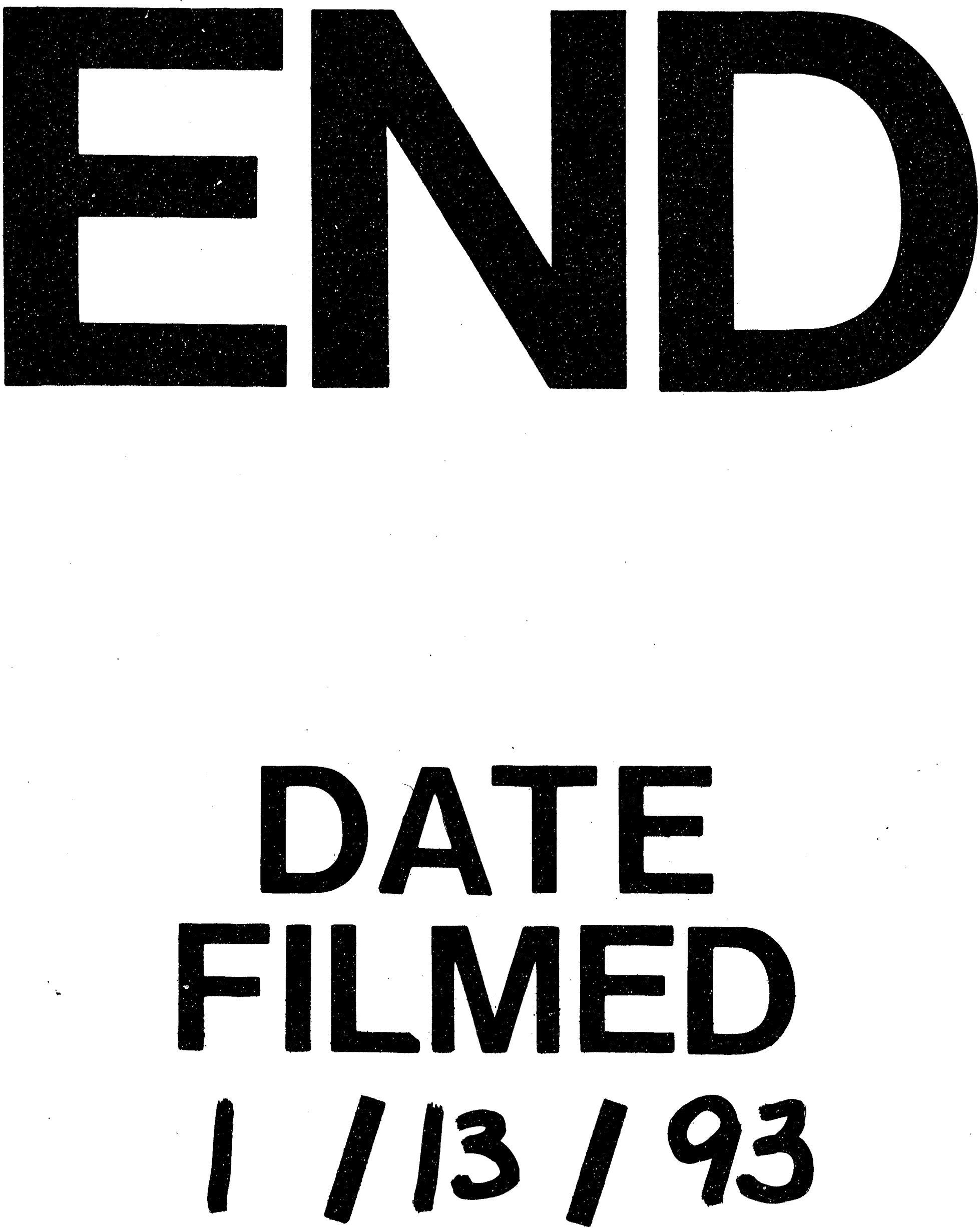
